\title{
Automation of control processes in the non- destructive testing units
}

\author{
Sergei Bekher ${ }^{1, *}$ and Andrei Kolomeets ${ }^{1}$ \\ ${ }^{1}$ Siberian Transport University, Dusi Kovalchuk st., 191, 630049, Novosibirsk, Russia
}

\begin{abstract}
The problems of automation of control processes in the nondestructive testing units are considered. The analysis of normative and technical documents is carried out and the main components of the nondestructive testing system and their parameters are determined. The developed software and relational database for monitoring and control of the unit have been integrated into trial operation in the non-destructive control units of the freight car repair company. Based on the statistical processing of control results, the possibility of assessing the efficiency of NDT inspectors and control devices was shown. The use of a network database and developed software makes it possible to improve the efficiency of control processes in the units of non-destructive testing by reducing the time spent on analysis and deciding on scheduled activities, the formation of reporting documentation.
\end{abstract}

\section{Introduction}

Reliability of technical means after manufacturing and repair largely depends on the integrity of the results of non-destructive testing of their elements. When operating freight cars in the period from 2003 to 2013, there were more than 14 breaks of the running parts of the cars, including 19 wrecks of rolling stock. Traces of fatigue defects are revealed at all breaks, which were not revealed during repair and operation [1]. The worst performance was recorded in 2013: 37 destructions of parts and 6 wrecks. Currently, the situation has improved by the efforts of JSC "Russian Railways", manufacturing plants, and car repair facilities. In 2016 the number of breaks has decreased more than 5 times and does not exceed 7.

The efficiency of the non-destructive control units is determined by the main components of the system: personnel, equipment, control conditions, technical documentation. One of the main tasks facing organizations involved in the manufacture, repair and operation of rolling stock is the fulfillment of the mandatory requirements of regulatory documentation for all components of the system at an arbitrary point of time [2]. The purpose of the study is to increase the efficiency of control of non-destructive testing units by reducing the time and ensuring the integrity of the initial data for decision-making when planning work.

\footnotetext{
*Corresponding author: beher@stu.ru
} 


\section{Method of research}

The adoption of organizational and technical solutions is based on the current state and the forecast of the development of the non-destructive testing system. To describe its state, systematic records are made. For example, the qualification of the personnel is characterized by the level of education, the date and place of professional development, work experience, skill category; control means - the year of manufacture, grade, date of check-out or calibration, etc. The largest amount of data (Figure 1) is contained in the information on control tools and monitoring reports, the total amount of data on the units of non-destructive testing can reach several thousand. Data of various types is used from the simplest logical (1/0) to complex text and binary ones, the size of which reaches several tens of megabytes. The efficiency and timeliness of decision-making under these conditions depends on the correct interpretation of the initial data [3]. To solve this problem, a network database and software have been developed. The program provides data collection, automated analysis and identification of critical states of the non-destructive testing system, planning of regular work, formation of a report on the activities of the units.

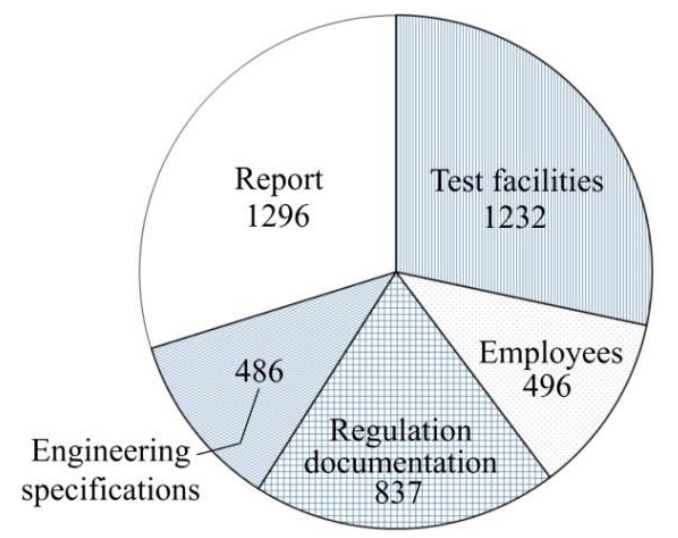

Fig. 1. The diagram of distribution of the total amount of data on the components of a non-destructive testing system.

For companies that include several enterprises, the priority is to ensure the unity of terminology. The different form of recording the same information significantly complicates the analysis of data on the activities of non-destructive testing units. Unification of terms and definitions is based on the results of trial operation of the software. Guides for basic concepts have been developed: methods and variants of control methods, testing means, organizations, parts, controlled zones and types of defects. Individual groups of concepts require revision over time, including when changing the regulatory documentation, so the program provides the ability to administer the tables of reference data.

When designing the database, the main components of the non-destructive testing system in the enterprise are identified and the relationships between them are described, presented in the form of an ER model in Figure 2 [4-5]. Most database tables are linked by "one-to-many" relationships. For example, a record in the "Devices" table is associated with several records about the control results in the "Results" table. The relationships between "many-to-many" tables are implemented using "one-to-many" intermediate relationships. 


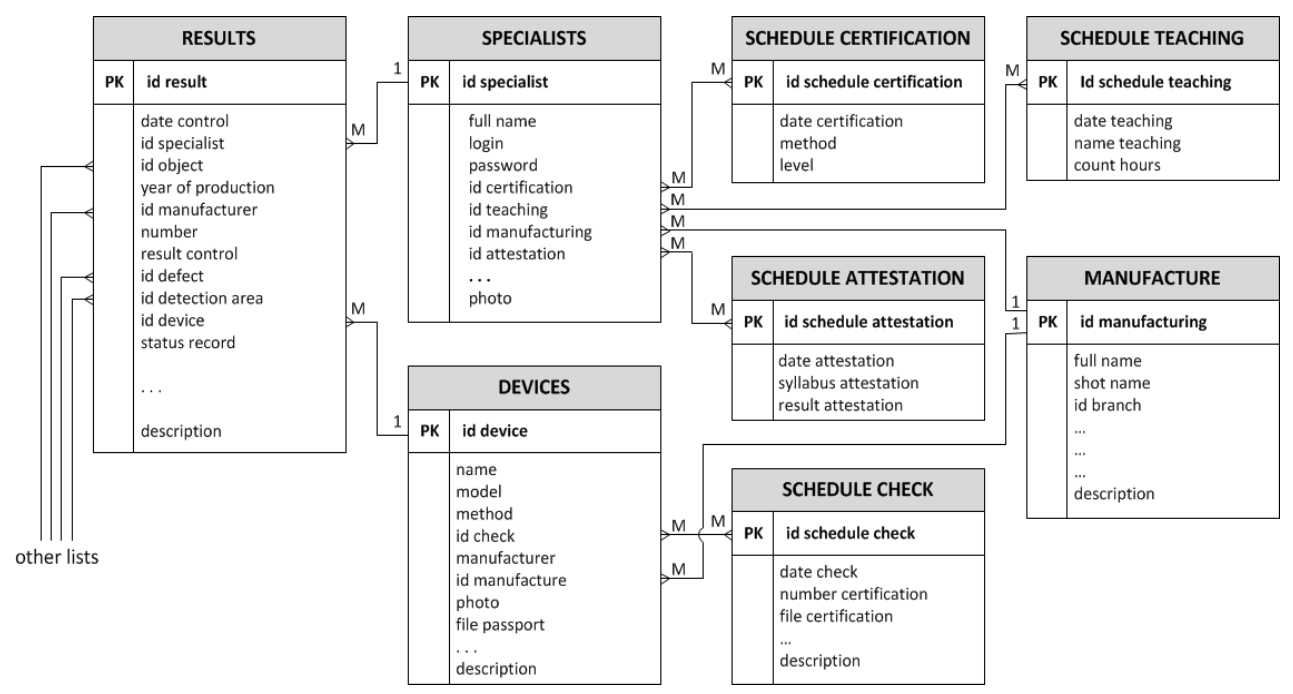

Fig. 2. Model of the relational database of the non-destructive testing units.

The use of the database has made it possible to reduce the processing time of primary data by automating the preparation of a report on the work of the unit [6-7]. The designed formats of stored data and the rules for their verification ensure the integrity of the database, and the use of guides reduces the amount of information that users input. At the same time, the time for performing these operations is reduced. The program implementation of the formation of reporting documents and statistical analysis of data will make it possible to release specialists in the process of preparation of systematic reports.

The advantage of a network database for the management of a company with several structural units is the operative obtaining of comparable and contrast information for each enterprise.

\section{Experimental data and results}

The units of non-destructive testing are obliged to ensure compliance with the mandatory requirements of regulatory documents at any time. A specified number of serviceable, verified or calibrated technical aids must be located at all control positions, and employees must undergo periodic qualification, certification and attestation in the workplace in accordance with the established procedure. The fulfillment of these conditions can be fully automated, and organizational decisions are made without the participation of the head of the unit. The program constantly displays a graphical image (Figure 3) of the current state and planned movements of control devices from the reserve to the working position, for verification or for repair, etc. The minimum required number of ultrasonic detectors in the wheel-roller shop is 2 units, and taking into account the need for verification or repair - 5 units (Figure 3) 


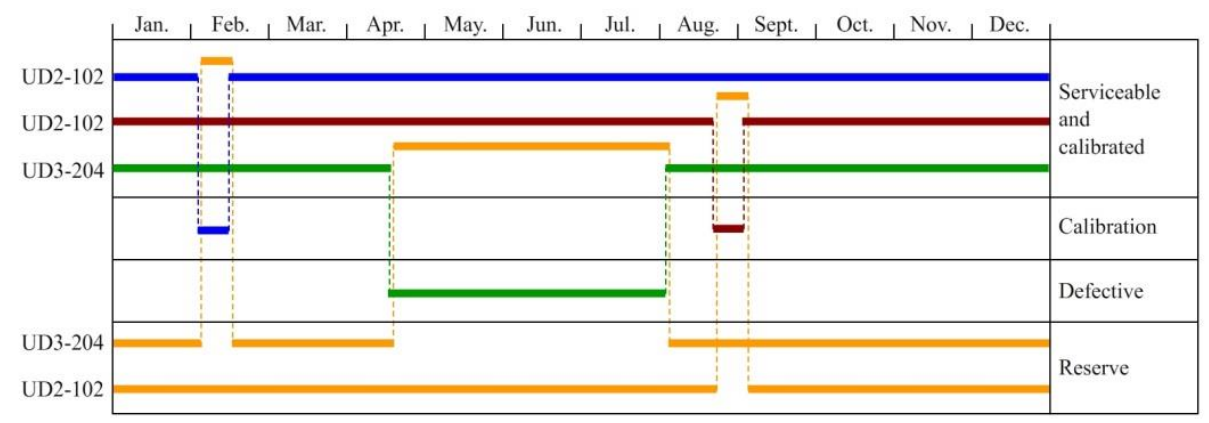

Fig. 3. Monitoring of the state of means of ultrasonic inspection of the wheel-roller shop and planning.

Statistical analysis of results of control allows real-time evaluation of the efficiency of each of the elements of the non-destructive testing system: workers, equipment and technical documentation. The relative number of bolsters (Figure 4) sent to repair by the results of non-destructive testing is $(50 \pm 1) \%$. To detect defects of this type (wear of inclined planes), special skills and work experience are not required, which is confirmed by a balancing distribution of the probability of their detection by employees of the enterprise.

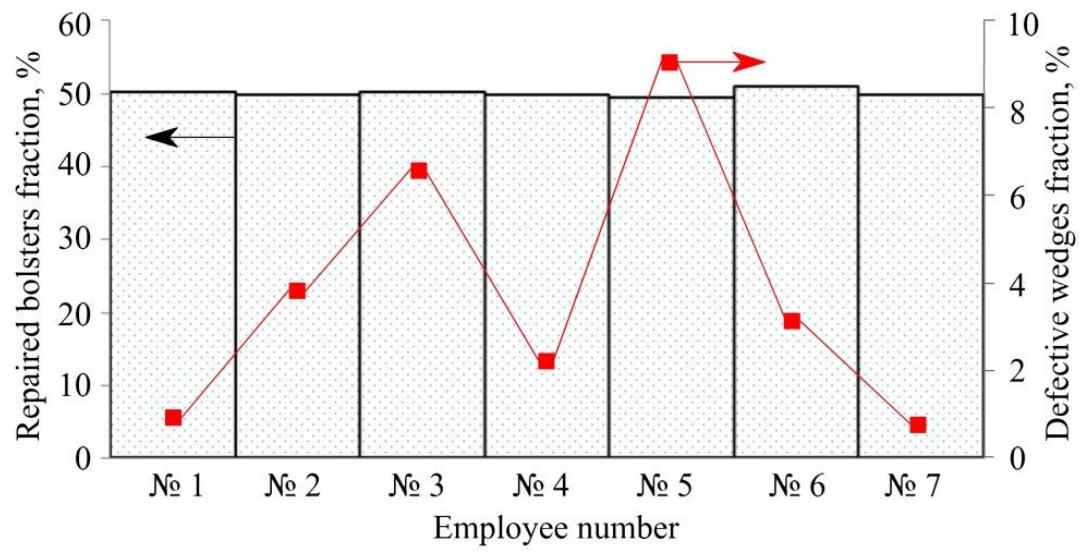

Fig. 4. Distribution of the proportion of bolsters and defective wedges of the traction yoke sent for the repair by the employees of the non-destructive testing unit.

On the other hand, to control the wedges of the traction yoke, the means of magnetic particle test are used [10-11]. Reliability of its results directly depends on the qualification of the operator. The lack of competence to identify false indications leads to an excessive rejection, and the violation of control technology - to the defect escape. The number of wedges of the traction yoke rejected by the employees varies in a wide range from 0.89 to $9 \%$. The amount of sampling is significant and consists of no less than 150 parts controlled by each employee within six months. To check the influence of the characteristics of the employee on the results of control, the dependence of proportion of rejected parts on the work experience is formed (Fig. 5). A rather high correlation coefficient of 0.93 is observed, indicating a strong connection between the results of control and work experience. To correct the existing imbalance in proportions of rejected parts by NDT inspectors with different length of service, corrective measures are required, including inspection and re-inspection, attestation of employees at the enterprise, and, if necessary, further training of employees in specialized training centers. 


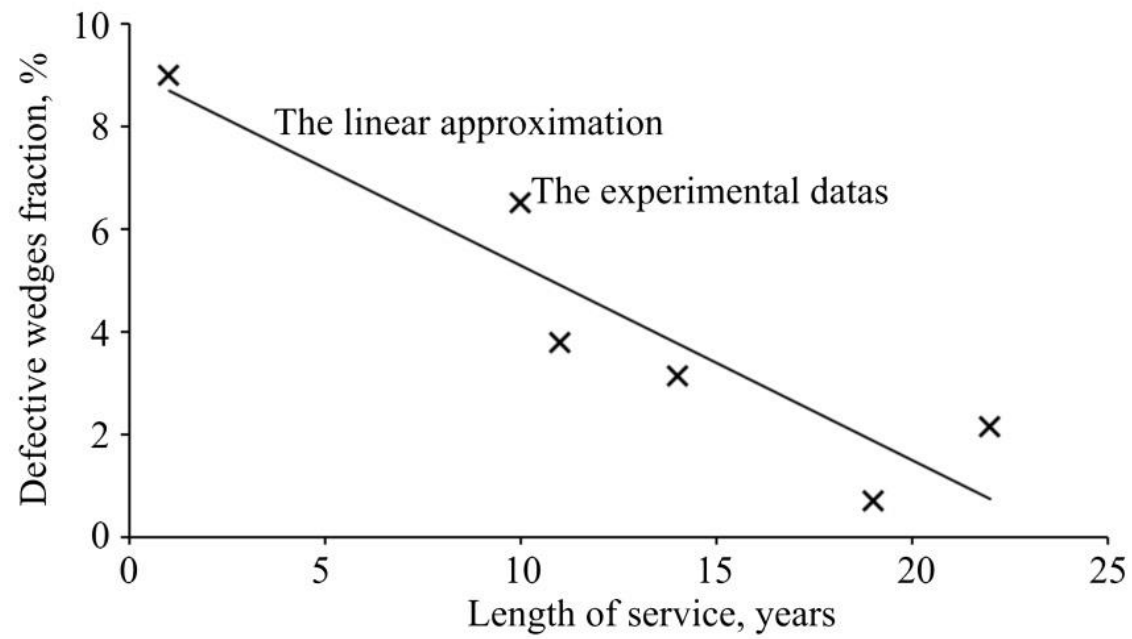

Fig. 5. Dependence of the relative number of rejected wedges of the traction yoke on the employee's length of service.

One of the most critical details, the destruction of which can lead to an accident or wreck of the rolling stock, are the side frames of freight cars [8-9]. Fatigue cracks in the corners of the pedestal jaw openings are the most dangerous and quite common defects. Magnetic particle detectors are used to detect them, and the internal surface of the parts is visually inspected. At car repair shops, the average level of rejection is $3.8 \%$ (Fig. 6). The results of the control carried out by the NDT inspector № 5 also have an extreme value of the rejection level and go beyond the confidence limits with a significance level of $10 \%$. The total amount of sampling is more than 4000 parts. An unusual but logical experimental fact is connected with the fact that the same employee demonstrates an extremely high rejection level on parts of one type and low on details of another type, which confirms the need for organizational measures.

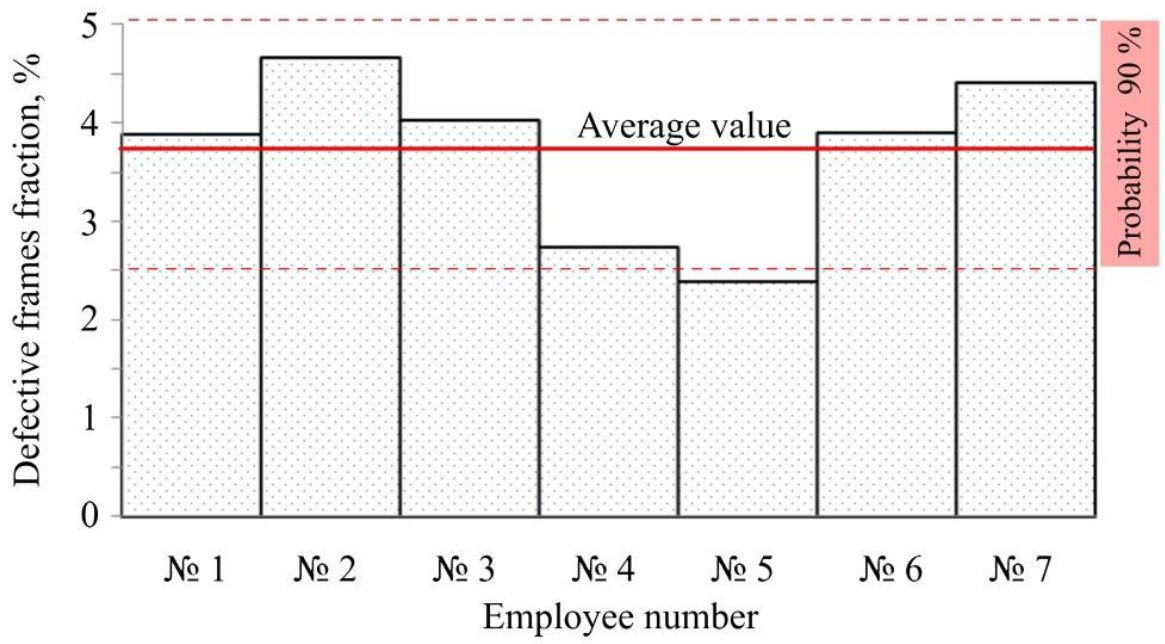

Fig. 6. Distribution of the proportion of rejected side frames to employees of the non-destructive testing unit, the average value and the confidence interval with a probability of $95 \%$. 


\section{Conclusion}

The developed software for the control of the non-destructive testing unit and monitoring of its activities is based on the use of relational databases. The program is implemented in the experimental operation in the car repair depots of the West Siberian Railway. Increasing the efficiency of the non-destructive testing system is achieved by reducing the time spent on organizational issues, the promptness of obtaining objective data on the status of the system components, ensuring uniformity and comparability of the results of work of several units of the same company.

Statistical analysis of the results of control provides opportunities for continuous monitoring of the competencies of the employees of the unit, the operability of control means and technical documentation. Based on the analysis of the results of the magnetic particle inspection of the side frames, bolsters and wedges of the traction yoke, it is shown that the proportion of the parts rejected by NDT inspector with 1 year of length of work does not correspond to the statistical distribution for the entire unit with a significance level of $10 \%$. It is recommended to carry out corrective measures to determine the causes and eliminate the imbalance of the results of control.

\section{References}

1. S.A. Bekher, A.L. Bobrov, A.A. Bolchanov, Vestnik of the Rostov State Transport University 2(42), 20-26 (2011)

2. A. Karen, Gudok, 212 (2017)

3. S. Roger, M. Bruce, Software Engineering: A Practitioner's Approach, 8th edit. (McGraw-Hill Education, 2014)

4. T. Bernhard, Entity-Relationship Modeling (Springer, 2014)

5. M. Ross, M. Stacia, Introducing Microsoft SQL Server 2012, 1st edit. (Microsoft Press, 2012)

6. L. Chao, Database Development and Management, 1st edit. (Auerbach Publications, 2006)

7. R.Raghu, G. Johannes, Database Management Systems, 2st edit. (McGraw-Hill College, 2000)

8. S.J. Kwon, Key Engineering Materials 321-323, 1483-1486 (2006)

9. K. Matsumoto, Materials Science Forum 571-572, 321-326 (2008)

10. V.V. Muravyev, O.V. Muravyeva, E.N. Kokorina, Russian Journal of Nondestructive Testing 49(1), 15-25 (2013)

11. V.V. Muravyev, L.V. Volkova, Russian Journal of Nondestructive Testing 49(9), 524529 (2013) 